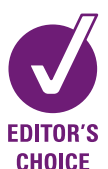

CHOICE

\title{
The impact of diabetes on the extent of pial collaterals in acute ischemic stroke patients
}

\author{
Marc A Lazzaro, ${ }^{1}$ Michael Chen, ${ }^{2}$ Gregory A Christoforidis, ${ }^{3}$ Yousef Mohammad ${ }^{2}$
}

${ }^{1}$ Froedtert Hospital and Medical College of Wisconsin,

Milwaukee, Wisconsin, USA

${ }^{2}$ Rush University Medical

Center, Chicago, Illinois, USA

${ }^{3}$ The University of Chicago,

Chicago, Illinois, USA

\section{Correspondence to}

Dr M A Lazzaro, Medical College

of Wisconsin, Department of

Neurology, 9200 West

Wisconsin Avenue, Milwaukee, WI 53226, USA;

mlazzaro2@gmail.com

Received 14 December 2010

Accepted 7 January 2011

Published Online First

1 February 2011

\section{ABSTRACT}

Introduction The threatened vascular territory in ischemic stroke is maintained by pial collateral circulation. Neovascularization varies among organ systems in diabetic patients. The effect of diabetes on the extent of pial collaterals is not well known. The pial collateral supply was compared among diabetic and non-diabetic patients presenting with acute ischemic stroke.

Material and methods Databases from two institutions were accessed to identify all patients who presented with acute middle cerebral artery occlusion and underwent emergent cerebral angiography. Angiograms were reviewed and graded using a previously published pial collateral grading system. The collateral grading system was dichotomized into good (pial grades 1-2) and poor (pial grades $3-5$ ). A $\chi^{2}$ analysis was performed to compare the association between diabetes and pial collateral grade.

Results 104 patients were identified; 56 (54\%) patients were men, mean age was 63.5 years and 22 (21\%) patients had diabetes. Mean admission National Institutes of Health Stroke Scale was 15.7. In the diabetic population, 15 (68.2\%) patients had good pial collaterals while seven (31.8\%) patients had poor pial collaterals. In the non-diabetic population, 59 (71.9\%) patients had good pial collaterals while 23 (28.0\%) patients had poor pial collaterals. There was no association between diabetes and pial collateral grade $\left(p=0.7290\right.$, Pearson $\left.\chi^{2}\right)$.

Conclusions This series suggests there is no association between diabetes and the extent of pial collaterals in ischemic stroke patients. Further studies are warranted to understand pial collateral patterns in acute ischemic stroke patients and their impact on clinical outcomes.

\section{INTRODUCTION}

Arterial occlusions in acute ischemic stroke result in complete cessation of blood flow thereby challenging the capacity of pial collateral circulation to maintain the threatened vascular territory. Limited reports describe angiographic characterization of pial collateral circulation in acute ischemic stroke patients. It has been shown that collateral circulation is an independent radiologic predictor of outcome after thrombolysis in acute ischemic stroke. ${ }^{1}$ In this study, retrograde filling to the M2 segment or distal mainstem of the middle cerebral artery (MCA), in comparison with other collateralization patterns, was predictive of a favorable outcome at 3 months. In the PROACT II study, angiography was used to assess recanalization and collateral circulation and several factors were found to be significantly associated with collateral supply. $^{2}$ Patients with full collateral supply had lower baseline National Institutes of Health Stroke Scale scores, smaller baseline CT infarct volumes and less cortical involvement. These studies suggest that pial collateral capacity is important in acute ischemic stroke. The factors that affect pial collaterals may have an important role in predicting outcome and selecting patients for treatment of acute ischemic stroke. One potential factor is diabetes. Neovascularization varies among organ systems in diabetics. Excessive angiogenesis occurs in the retina while impaired angiogenesis occurs in the coronary and peripheral vasculature. Peripheral artery disease is more than twice as common in diabetic patients compared with non-diabetic patients, ${ }^{3}$ and coronary collateral artery development is poor in diabetic patients. ${ }^{4}$ Although the effect of diabetes has been recognized in other vascular systems, its impact on the extent of pial collaterals in cerebral artery disease is not well known. In this study, we sought to compare the pial collateral supply among diabetic and nondiabetic patients presenting with acute ischemic stroke.

\section{MATERIALS AND METHODS}

We performed a retrospective analysis. Databases from two institutions: Rush University Medical Center, Chicago, Illinois and Ohio State University, Columbus, Ohio, were accessed. These databases included acute ischemic stroke patients who underwent emergent catheter angiography. To be included in the analysis, all patients had an acute ischemic stroke, underwent emergent catheter angiography and had a proximal MCA occlusion. All other sites of vessel occlusion were excluded. Clinical and demographic data were abstracted from charts. Cerebral angiograms were reviewed on a picture archiving and communications system. Angiograms were interpreted by a single experienced angiographer at each center who was blinded to the clinical data.

Angiograms were reviewed and graded using a pial collateral grading system previously published by Christoforidis and colleagues. ${ }^{5}$ This pial collateral grading system relies on retrograde contrast opacification of the MCA territory on delayed angiogram images. Retrograde flow into the MCA territory is divided into five grades (figures 1 and 2).

Blood flow through the anterior cerebral artery passes through the pial collateral vessels filling the MCA territory in a retrograde fashion. A grade 5 is assigned if there is little or no significant reconstitution of the territory of the occluded vessel. Grade 4 is assigned if there is collateral reconstitution of 


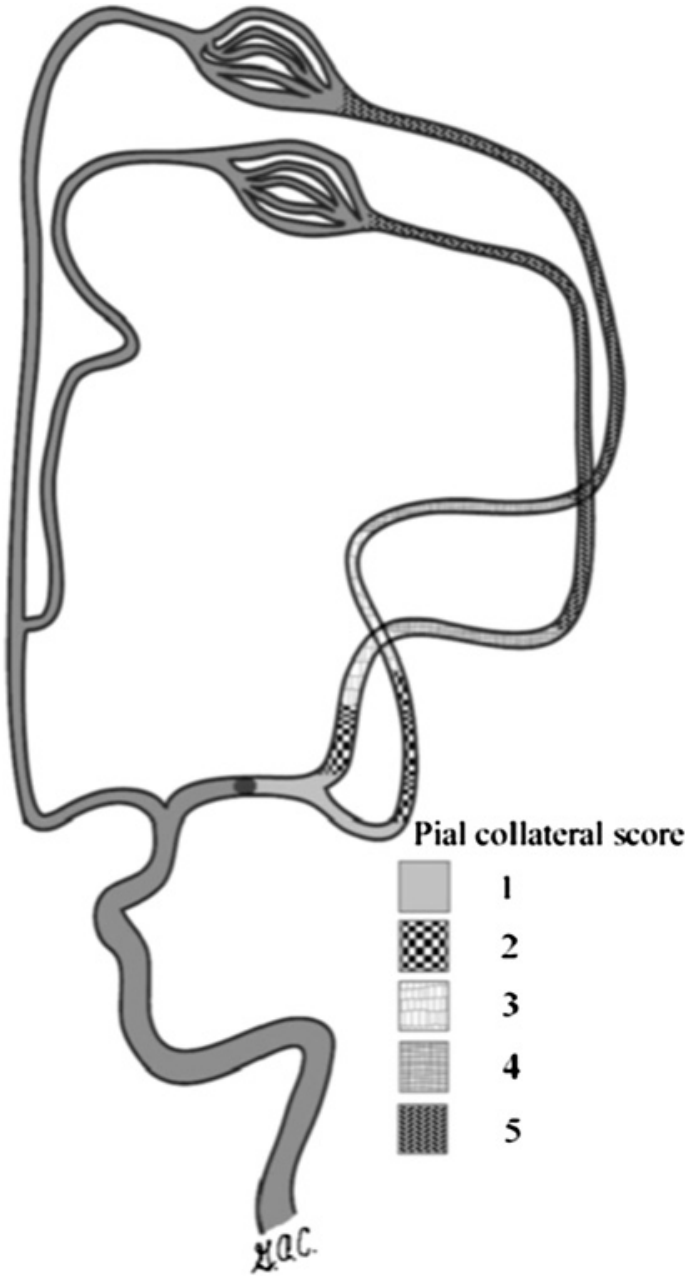

Figure 1 Schematic diagram of the pial collateral grading system. Reproduced with permission from Christoforidis and colleagues. ${ }^{5}$

vessels two segments distal to the occluded vessel (ie, if there is reconstitution up to the $M 3$ segment branches). Grade 3 is assigned if the reconstitution reaches the distal portion of the segment adjacent to the occluded vessel. Grade 2 shows reconstitution of the proximal portion of the segment adjacent to the occluded vessel (ie, proximal reconstitution of the M2 branch). Grade 1 is assigned for collateral reconstitution to the distal portion of the occluded vessel segment.

The collateral grading system was further dichotomized into 'good' and 'poor' grades. A 'good' grade included pial grades of $1-2$ while a 'poor' grade included pial grades of $3-5$. Statistical analysis was performed with Pearson's $\chi^{2}$ test using JMP7 software (SAS Institute). The study was approved by the institutional review board prior to collection of the data.

\section{RESULTS}

We identified 104 patients who met the inclusion criteria for analysis. Baseline characteristics are summarized in table 1.

In this series of patients, $56(54 \%)$ were men and $22(21 \%)$ patients had diabetes. All patients had a proximal MCA occlusion and these were nearly evenly divided between left and right MCAs (62 (59.6\%) vs 42 (40.4\%), respectively). Mean age was 63.5 years (SD 13.6). The patients in this series had moderate to severe stroke, as evidenced by the mean National Institutes of Health Stroke Scale score of 15.7 (SD 5.0). Mean time to angiography was $266 \mathrm{~min}$ (SD 90). Distribution of cases by time to angiography revealed that the majority of cases were treated between 2 and $6 \mathrm{~h}$ from symptom onset $(\mathrm{n}=86,82.7 \%$ ) (table 2 ).

In the diabetic population, 15 (68.2\%) patients had good pial collaterals while seven (31.8\%) patients had poor pial collaterals. In the non-diabetic population, 59 (71.9\%) patients had good pial collaterals while $23(28.0 \%)$ patients had poor pial collaterals (table 3).

\section{DISCUSSION}

This series suggests there is no association between diabetes and the extent of pial collaterals in ischemic stroke patients with large artery occlusions. Although the sample size was small, there were a sufficient number of patients to detect a twofold difference with $80 \%$ power.

This finding is contrary to observations of collateral circulation in other organs. The effect of diabetes on collateral supply
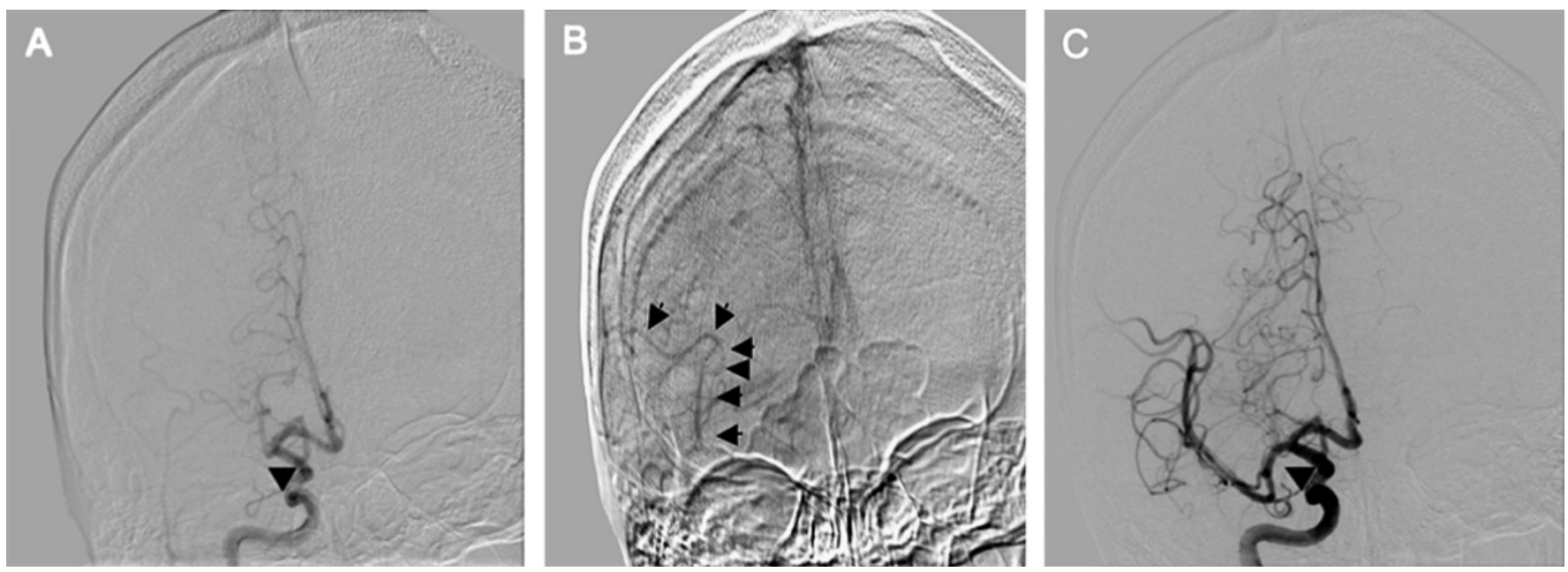

Figure 2 Illustration of the use of the pial collateral grading system. An arterial phase anteroposterior cerebral angiogram from a right internal carotid artery (ICA) injection shows opacification of the right ICA, anterior cerebral artery and an abrupt cut-off (arrow) of the proximal portion of the right middle cerebral artery (MCA) (A). The venous phase of the same angiographic run demonstrates reconstitution of the right MCA (arrows) with opacification extending into the proximal portion of the segment adjacent to the occluded vessel, or the M2 branch in this M1 occlusion (B). This is assigned a grade 2. Following recanalization, robust flow is evident through the right MCA at the site of the prior occlusion $(C$, arrow). Arterial phase opacification of the MCA branches indicate antegrade filling. 
Table 1 Patient characteristics $(n=104)$

\begin{tabular}{lc}
\hline Characteristic & \\
\hline Men (n (\%)) & $56(54)$ \\
Diabetes (n (\%)) & $22(21)$ \\
Left middle cerebral artery (n (\%)) & $62(59.6)$ \\
Age (years) (mean (SD)) & $63.5( \pm 13.6)$ \\
Admission NIHSS (mean (SD)) & $15.7( \pm 5.0)$ \\
\hline
\end{tabular}

NIHSS, National Institutes of Health Stroke Scale.

varies among different organ systems leading to either impaired or excessive neovascularization. The mechanism of these effects is not clear. The impact of diabetes on the cerebral vasculature, as measured by angiography, has not previously been described. In an animal model, neovascularization was measured in control rats compared with type 2 diabetic rats after MCA occlusion. ${ }^{6}$ Diabetic rats had augmented neovascularization and developed smaller infarct size and greater edema. Both angiogenesis and arteriogenesis were observed, suggesting the adaptive mechanisms are capillary sprouting and remodeling of native collaterals into functional arterioles. The model however included an in-bred strain of rats that were young and therefore had only a short duration of diabetes.

There are limited reports describing angiographic pial collateral characteristics in humans. Understanding the impact of diabetes on pial collateral supply may be helpful for predicting outcomes, selecting patients for recanalization therapies and controlling for pial collateral differences in clinical trial design. This small series suggests that a twofold or greater difference in pial collateral supply does not exist between diabetic and nondiabetic ischemic stroke patients with large artery occlusions but further study with larger series and alternative grading systems may identify a difference.

We chose to use a pial collateral grading system that is based on catheter angiography because it offers spatial and temporal resolution as well as retrograde flow characterization, which may be superior to alternative modalities such as CT or MR angiography. The grading system was dichotomized in the present study because a previous report showed a correlation between the dichotomized pial collateral score and outcome in patients who had complete recanalization. ${ }^{5}$ The grading system was dichotomized in the same way, grades 1 and 2 as 'good' and grades $3-5$ as 'poor'. Those patients with a pial grade of 1 or 2 were more likely to have a discharge modified Rankin score of $<3$ compared with patients with a pial collateral grade of $3-5$ $(\mathrm{p}=0.013)$. Only MCA occlusions were included in this analysis. This was necessary to use the chosen grading scale and furthermore offers a more homogenous series of infarcts. In using only MCA occlusions, the anterior cerebral artery-middle cerebral artery collateral bed is isolated, allowing for study of a single vascular bed. This grading scale also minimizes collateral contamination from the posterior circulation or contralateral circulation as it is based on a carotid injection.

Table 2 Time to angiography $(n=104)$

\begin{tabular}{lcccccc}
\hline & & \multicolumn{2}{l}{ Pial grade } & & \multicolumn{2}{c}{ Presence of DM } \\
Time $(\boldsymbol{m i n})$ & Cases $(\mathbf{n}(\%))$ & Grades 1, 2 & Grades 3-5 & & DM & Non-DM \\
\hline$<120$ & $9(8.7)$ & 6 & 3 & 3 & 6 \\
$121-240$ & $29(27.9)$ & 20 & 9 & 5 & 24 \\
$241-360$ & $57(54.5)$ & 40 & 17 & 11 & 46 \\
$>360$ & $8(7.7)$ & 6 & 2 & 2 & 6 \\
\hline
\end{tabular}

$\mathrm{DM}$, diabetes mellitus.
Table 3 Pial collateral grades

\begin{tabular}{lcll}
\hline & Diabetic $(\mathbf{n}=22)$ & Non-diabetic $(\mathbf{n}=\mathbf{8 2})$ & $\mathbf{p}$ Value \\
\hline Good (grades 1, 2) & $15(68.2 \%)$ & $59(71.9 \%)$ & 0.734 \\
Poor (grades 3-5) & $7(31.8 \%)$ & $23(28.0 \%)$ & 0.459 \\
\hline
\end{tabular}

There was no association between diabetes and pial collateral grade $(p=0.7290$, Pearson's $\left.\chi^{2}\right)$.

There are several limitations to this study. This was a small, retrospective series that was powered only to detect a considerable difference in pial collateral grades. The grading system used was one of many that are available for evaluation of pial collateral circulation and other scales may allow comparison of additional characteristics such as vessel filling time. This grading system has been used in only a limited number of publications and has not been rigorously tested for inter-rater reliability. Markers of longstanding poorly controlled diabetes such as hemoglobin A1C levels, retinopathy and renal dysfunction were not available for these patients. Duration of diabetes and diabetic control may have varied considerably among patients and contributed to differences in pial collateral capacity.

Future studies will be needed to further evaluate characteristics of pial collaterals and factors that impact collateral supply. An alternative collateral flow grading system proposed by the ASITN/SIR includes characterization of flow as 'slow' or 'rapid', ' elements that are not defined in the grading system used in the present series and which may be important in assessing factors that impact pial collaterals. Angiographic collateral flow has been shown to be independent of perfusion mismatch in predicting infarct size ${ }^{8}$ but the two may be complimentary and future studies should include further investigation of these factors as predictors of outcome. Collateral circulation is complex and factors affecting the extent of collateralization is likely multifactorial. Understanding the factors that affect pial collaterals may guide future therapies for enhancing pial collaterals, improve patient selection for thrombolysis and reduce hemorrhage from recanalization.

\section{CONCLUSIONS}

This study did not detect a twofold or greater difference in angiographic pial collateralization between diabetic and nondiabetic acute ischemic stroke patients with a large artery occlusion, suggesting there is no difference in pial collateralization between the two groups. The pial collateral grading system is a useful technique for evaluating pial collateral vessels. Further study is needed to understand factors affecting pial collateral

\section{Key messages}

- Pial collateral vessels serve an important role in sustaining the brain tissue at risk during large artery occlusion but there is a limited understanding of the factors that may affect pial collaterals.

- Neovascularization varies among organ systems in diabetics, resulting in excessive angiogenesis in the retina and impaired development in the coronary and peripheral vascular systems but the impact of diabetes on pial collaterals has not been described.

- This series suggests that there is no large difference in pial collaterals between diabetics and non-diabetics in large artery acute ischemic stroke patients. 
patterns in acute ischemic stroke patients and their impact on clinical outcomes.

\section{Competing interests None.}

Ethics approval This study was conducted with the approval of the Rush University Medical Center Institutional Review Board and Ohio State University Institutional Review Board.

Provenance and peer review Not commissioned; externally peer reviewed.

\section{REFERENCES}

1. Kucinski T, Koch C, Eckert B, et al. Collateral circulation is an independent radiological predictor of outcome after thrombolysis in acute ischaemic stroke. Neuroradiology 2003; $45: 11-18$

2. Roberts HC, Dillon WP, Furlan AJ, et al. Computed tomographic findings in patients undergoing intra-arterial thrombolysis for acute ischemic stroke due to middle cerebral artery occlusion: results from the PROACT II trial. Stroke 2002;33:1557-65.
3. Selvin E, Erlinger TP. Prevalence of and risk factors for peripheral arterial disease in the United States: results from the National Health and Nutrition Examination Survey, 1999-2000. Circulation 2004;110:738-43.

4. Abaci A, Oguzhan A, Kahraman S, et al. Effect of diabetes mellitus on formation of coronary collateral vessels. Circulation 1999;99:2239-42.

5. Christoforidis GA, Mohammad Y, Kehagias D, et al. Angiographic assessment of pia collaterals as a prognostic indicator following intra-arterial thrombolysis for acute ischemic stroke. AJNR Am J Neuroradiol 2005;26:1789-97.

6. Li W. Prakash R, Kelly-Cobbs Al, et al. Adaptive cerebral neovascularization in a model of type 2 diabetes: relevance to focal cerebral ischemia. Diabetes 2010;59:228-35.

7. Higashida RT, Furlan AJ, Roberts $\mathrm{H}$, et al; Technology Assessment Committee of the American Society of Interventional and Therapeutic Neuroradiology; Technology Assessment Committee of the Society of Interventional Radiology. Trial design and reporting standards for intra-arterial cerebral thrombolysis for acute ischemic stroke. Stroke 2003; 34:e109-37.

8. Bang OY, Saver JL, Buck BH, et al; UCLA Collateral Investigators. Impact of collatera flow on tissue fate in acute ischaemic stroke. J Neurol Neurosurg Psychiatry 2008;79:625-9

\section{Correction}

(C) 2011 by the American College of Cardiology Foundation

In the article by Brott TG, Halperin JL, Abbara S, et al. 2011ASA/ACCF/AHA/AANN/AANS/ ACR/ASNR/CNS/SAIP/SCAI/SIR/SNIS/SVM/SVS Guideline on the Management of Patients With Extracranial Carotid and Vertebral Artery Disease: Executive Summary: A Report of the American College of Cardiology Foundation/American Heart Association Task Force on Practice Guidelines, and the American Stroke Association, American Association of Neuroscience Nurses, American Association of Neurological Surgeons, American College of Radiology, American Society of Neuroradiology, Congress of Neurological Surgeons, Society of Atherosclerosis Imaging and Prevention, Society for Cardiovascular Angiography and Interventions, Society of Interventional Radiology, Society of NeuroInterventional Surgery, Society for Vascular Medicine, and Society for Vascular Surgery, which appeared February 22, 2011 issue of the Journal of American College of Cardiology (J Am Coll Cardiol 2011;57:1002-44; doi:10.1016/j. jacc.2010.11.005), and June 2011 of the Journal of NeuroInterventional Surgery (J NeuroIntervent Surg 2011;3:100-30), the following corrections are necessary:

In Section 18, "Recommendations for Management of Patients With Cervical Artery Dissection," the Class IIa Recommendation \#1 (p. 1012), which begins "For patients with symptomatic cervical artery dissection, anticoagulation with...," should be changed to read: 1. Antithrombotic treatment with either an anticoagulant (heparin, low molecular weight heparin, or warfarin*) or a platelet inhibitor (aspirin, clopidogrel, or the combination of extended-release dipyridamole plus aspirin*) for at least 3 to 6 months is reasonable for patients with extracranial carotid or vertebral arterial dissection associated with ischemic stroke or TIA $(72 \mathrm{a}-72 \mathrm{~d})$. (Level of Evidence B)

*Drugs are not listed in order of preference.

The following references should be added to the reference list:

72a. Metso TM, Metso AJ, Helenius J, et al. Prognosis and safety of anticoagulation in intracranial artery dissections in adults. Stroke 2007;38:1837-42.

72b. Engelter ST, Brandt T, Debette S, et al; for the Cervical Artery Dissection in Ischemic Stroke Patients (CADISP) Study Group. Antiplatelets versus anticoagulation in cervical artery dissection. Stroke 2007;38:2605-11.

72c. Menon R, Kerry S, Norris JW, Markus HS. Treatment of cervical artery dissection: a systematic review and meta-analysis. J Neurol Neurosurg Psychiatry 2008;79:1122-7.

$72 \mathrm{~d}$. Georgiadis D, Arnold M, von Buedingen HC, et al. Aspirin vs anticoagulation in carotid artery dissection: a study of 298 patients. Neurology 2009;72:1810-5.

J Neurolntervent Surg 2011;3:245. doi:10.1136/jnis.2011.004762corr1 